\title{
The Role of MiR-124 in Bladder Cancer - A Review of the Literature
}

\author{
Costin Petcu ${ }^{1,2}$, Catalin Baston ${ }^{1,2 *}$, Emil Angelescu ${ }^{1,2}$, Maria Mirela Iacob ${ }^{3}$, Ileana \\ Constantinescu $^{3,4}$, Ioanel Sinescu ${ }^{1,2}$
}

\section{Urological Surgery Department, "Carol Davila” University of Medicine and Pharmacy, Romania}

2. Center of Uronephrology and Kidney Transplant, Fundeni Clinical Institute, Romania

3. Center of Immunogenetics and Virology, Fundeni Clinical Institute, Romania

4. Department for Immunogenetics and Virology, "Carol Davila” University of Medicine and

Pharmacy, Romania

\begin{abstract}
MicroRNAs (miRNAs) are a group of non-coding RNA molecules that have an important role in modulating the expression of genes involved in regulating cellular functions. A growing number of studies suggest the abnormal expression of microRNAs in different types of cancer cells. MiRNA-124 is a microRNA that is down-regulated in many types of cancer cells, including bladder cancer. Our objective is to provide a review of the key publications that studied the effect of miR-124 on bladder cancer. This review focus on the targets and different pathways of miR-124 that were identified in various studies and differences between their expressions in normal urothelium and tumor tissues. We also include data regarding urinary methylations levels of miR-124 and their role in bladder cancer diagnosis and prognosis. Subsequently, we establish future perspectives of miR-124 research and its promising role in bladder cancer.
\end{abstract}

Keywords: bladder cancer, miR-124, microRNA, miR-124 target, miRNAs.

Received: 30 th June 2020; Accepted: 19 $9^{\text {th }}$ November 2020; Published: $9^{\text {th }}$ December 2020

Bladder cancer is the fifth most common malignancy in Europe with an incidence of 197,105 new cases in 2018 and the second most common urological neoplasm after prostate cancer. It is responsible for approximately 65,000 deaths per year in Europe according to the International Agency for Research on Cancer [1]. The same agency estimated that bladder cancer had an incidence of approximately 550,000 new cases worldwide in 2018, while 200,000 people died in 2018 because of this type of cancer. The worldwide 5-year prevalence reaches 1,650,000 cases, which explains the important economic impact that bladder cancer has on health systems around the globe [1].

\footnotetext{
" Corresponding author: Catalin Baston, Center of Uronephrology and Kidney Transplant, Fundeni Clinical Institute, Bucharest, Romania. E-mail: urologiefundeni@yahoo.com
} 
The majority of bladder cancers are histologically represented by urothelial carcinomas, which are accountable for almost $95 \%$ of the cases. Squamous cell carcinoma causes approximately $3 \%$ of bladder cancer cases worldwide and is associated with chronic inflammation of the urinary tract [2]. Several countries in Africa and the Middle East have the majority of cases with squamous cell carcinoma histology due to schistosomiasis, an endemic infection caused by a trematode. Adenocarcinomas, the third most frequent histological type of bladder cancer, can be found in approximately $1 \%$ of cases [2].

Available data show that the vast majority of human DNA sequences are actively transcribed but less than $3 \%$ of them encode a protein, while non-coding RNAs represent the major part of transcripts. MicroRNAs (miRNAs) are a group of non-coding RNA molecules that include 1825 nucleotides and play an important role in modulating the expression of genes involved in regulating cellular functions like proliferation, migration, invasion or apoptosis, by attaching to the 3'-untranslated region of targeted messenger RNA (mRNA). MiRNAs attach to the complementary recognition sequences of mRNAs and determine their degradation, inhibition and finally suppressing the gene that was targeted by the mRNA.

MiRNA-124 was initially detected in mice [3] and further studies found that human miRNA-124 has abnormal expressions in many types of cancers. MiR-124 has three different gene sequences that can be processed from three different locations: miR-124a-1 located at chromosome 8p23.1, miR-124a-2 located at chromosome $8 \mathrm{q} 12.3$ and miR-124a-3 located at 20q13.33 [4]. MiR-124 is one of the most studied microRNAs with proven interactions with many types of cancers, including lung cancer, colorectal cancer, leukemia and gastric cancer [5-9]. Even if the majority of articles conclude that miR-124 has a tumor-suppressive role in relation to different types of cancers, it can also have an oncogenic effect in patients with cholangiocarcinoma or acute lymphoblastic leukemia [10]. The effect of miR-124 in bladder cancer has been studied in several articles published in the last decade. The proven impact of miR-124 in bladder cancer propelled it as one of the most important miRNAs involved in bladder cancer and, consequently, more research papers regarding the molecular pathway and the actions of miR-124 have been published in the last 3-4 years.

The aim of this article is to provide a review of the key publications about miRNA-124 and its role in patients with bladder cancer. We included studies that we accessed via PubMed Central, PMC Europe, and Scopus. We used "bladder cancer", "urothelial carcinoma", "miRNA-124", "miR-124" and "microRNA-124" as keywords for our search of literature databases. We selected 16 articles containing original research matching our criteria that studied the effect of miR-124 in bladder cancer. Fourteen selected articles studied the expressions of miR-124 determined in tissues, while two of them used urinary expressions of miR-124 to evaluate its capability to act as a diagnostic or cancer progression biomarker. Multiple studies that have been published, investigate the correlations between tissue miRNA expression or urinary miRNA methylation and bladder cancer.

The vast majority of authors used tumor tissue and normal adjacent tissue samples collected from patients with bladder cancer. Quantitative real-time polymerase chain reaction (RT-qP$\mathrm{CR}$ ) and Western blot analysis were performed to determine the expression of miR-124 in both tumor and normal urothelium samples. Moreover, most of the researchers also used normal bladder urothelium cell lines and bladder cancer cell lines that were transfected with miR-124 inhibitors, miR-124 mimics, target siRNA (small interfering/ silencing RNA) or target cDNA (complementary DNA) to compare results with 
the normal and tumor tissues obtained from the bladder cancer patients that suffered the same process.

One study that was published in 2018 investigated the relationship between miR-124-3p and DNA methyltransferase 3B (DNMT3B) in 84 patients with bladder cancer [11]. The researchers used normal bladder urothelium cell line (SV-HUC-1) and bladder cancer lines (T24, 5637, RT4) together with normal and tumor tissues collected from bladder cancer patients. The authors concluded that bladder cancer tissue had a lower expression of miR-124-3p than normal tissue. Controversially, analysis of malignant cell lines showed that miR-124-3p had higher levels in the most invasive cell line (T24) and the lowest levels in RT4 low grade bladder cancer cell line, while DNMT3B was overexpressed in RT4 cell line comparing to T24 cell line. Higher expression of miR-124-3p can lower the proliferation rate, suppress invasion and promote apoptosis in tumor cells by suppressing DNMT3B, concluding that miR-124-3p has a tumor suppressive function in bladder cancer cells. Moreover, DNMT3B is an important promoter of bladder cancer invasion, proliferation and its expression was inhibited by miR-124-3p [11]. Zhang et al. [12] studied the connection between miR-124 and cyclin-dependent kinase (CDK4) gene by transferring different types of bladder cancer cell lines, transfected or not with miR124 , to lab mice. They used four different types of bladder cancer cell lines (HT1197, HT1376, J82, 5637) and normal urothelium cell lines (HBEC) along with tumor tissue samples obtained from 27 bladder cancer patients. The authors did not include adjacent normal bladder tissue collected from the bladder cancer patients in their study. Their conclusion was that miR-124 could target CDK4 by binding to its 3'-untranslated region. Analysis of bladder cell lines noted that levels of miR-124 were inversely correlated with CDK4, while HT1197 cell line had the highest levels of
CDK4 and lowest expression of miR-124. CDK4 was over-expressed in the bladder cancer tissue while miR-124 was down-regulated in human tumor tissue. The tumor cell proliferation was significantly lower in tumor tissue of lab mice, transfected with miR-124. The outcomes firmly suggest that miR-124 suppresses bladder tumor progression by targeting CDK4 in vitro and in vivo [12].

A study lead by Cao et al. that was published in 2019 [13] used pairs of bladder cancer tissue and adjacent normal urothelium obtained from 83 bladder cancer patients together with bladder cancer cell lines (T24, 5637, J82, UM-UC-3), normal urothelium cells (SV-HUC-1) and human embryonic kidney cell lines (HEK293). MiR-124 expression was down-regulated in all bladder cancer cell lines compared with normal urothelium cell line, while the lowest levels of miR-124 were observed in T24 and 5637 cell lines. The authors also concluded that miR-124 directly targeted CDK4 and inhibited cell proliferation and viability. It was noticed that over-expression of miR-124 led to down-regulation of CDK4, vascular endothelial growth factor (VEGF), E2F3 and Ki-67. However, all these changes were cancelled by CDK4 over-expression [13].

Aurora kinase A (AURKA) is an enzyme with an important role in cell division, during meiosis and mitosis. It seems that AURKA modulates the development of microtubules and helps the stability of the spindle pole in the course of chromosome segregation. Over-expression of AURKA is linked with poor prognosis because it contributes to oncogenesis and this gene can be regulated by different miRNAs in different kinds of cancers. For instance, AURKA is down-regulated by tumor suppressor miR-199a-3p in patients with prostate cancer [14]. In a study that used bladder cancer specimens and paired adjacent normal urothelium obtained from 36 patients treated by radical cystectomy, togeth- 
er with normal bladder tissue (SV-HUC-1) and urothelial tumor cell lines (5637, T24, RT4, J82), bladder cancer cell lines had miR-124-3p under-expressed but also over-expression of AURKA mRNA compared with normal cell lines [15]. The same study shows remarkably lower mRNA and protein expression of AURKA than the control group after transfection of miR-124$3 p$ and concludes that miR-124-3p can directly suppress AURKA and can inhibit cell migration, proliferation and induce apoptosis by regulating AURKA [15].

$\mathrm{X}$-inactive specific transcript (XIST) is a long non-coding RNA (lncRNA) that has a proven pathological role in different types of cancers. Androgen receptor (AR) is a ligand-activated transcriptional factor which modulates the biological role of androgen. It plays an important role in developing and progression of prostate, kidney, breast and bladder cancer. AR over-expression is correlated with disease progression and can also contribute to invasion and metastasis of bladder cancer [16]. One study used 67 pairs of tumor tissue and adjacent normal tissue obtained from bladder cancer patients, along with 4 types of bladder cancer cell lines (TCCSUP, EJ, EW780, UM-UC-3) and normal urothelium cell line (SV-HUC-1) that were transfected with miR-124 mimics or miR-124 inhibitor [17]. Both XIST and AR were over-expressed in tumor tissue and in all bladder cancer cell lines, compared with adjacent tissue and with SV-HUC-1 cell line. Moreover, higher expression of XIST and $\mathrm{AR}$ in tissues collected from bladder cancer patients were correlated with poorer bladder cancer stage and poorer prognosis. There might also be a connection between XIST and AR - some data suggest that XIST affects bladder cancer growth and metastasis by modulating AR expression. The authors conclude that XIST represents a competing endogenous RNA (ceRNA) for miR124 , thus inhibiting its expression through direct binding [17].
Integrins have an important role in survival, proliferation, migration and invasion in normal and malignant cells because they are the most important receptors that help cells adhere to extra-cellular matrix. In a study that used bladder cancer tissue and adjacent normal urothelium obtained from 36 patients, along with bladder cancer cell lines (T24, 5637, J82) and normal bladder urothelium (SV-HUC-1), integrin $\alpha 3$ (ITGA3) was found to be up-regulated in bladder cancer tissue compared with normal tissue [18]. Regarding the analysis of bladder cell lines, miR-124 levels were lower and ITGA3 levels were higher in all three bladder cancer cell lines compared with SV-HUC-1 cell line, while ITGA3 expression level was 2-3 times higher in malignant cell lines than normal. This study demonstrated that miR-124-3p could target ITGA3 by directly lowering its level in tumor tissue while migration and invasion of bladder cancer cells was suppressed in tissues with forced expression of miR-124-3p. Silencing ITGA3 decreased tumor cell viability and proliferation and flow cytometry showed higher apoptosis rate after the cells were transfected with silencing ITGA3 [18].

The E3 ubiquitin ligase ubiquitin-like with PHD and RING finger domain 1 (UHRF1) has an important contribution to DNA methylation during replication, serving as a substantial regulator of epigenetic modification and binding to methylated promoters of cancer-suppressing genes by retrieving DNA methyltransferase 1 . It was observed that miR-124 suppresses UHRF1 expression by targeting its binding sites and also targeting UHRF1 transcription, in a study that used tumor tissue and normal urothelium cells obtained from bladder cancer patients together with urothelial cancer cell lines (J82, T24), human embryonic kidney cell lines (HEK 293) and normal urothelium cell lines (SV-HUC-1) [19]. An inverse relation between miR-124 and UHRF1 was observed in both bladder cancer tissue (where UHRF1 was over-expressed) and 
in normal bladder tissue (where miR-124 was over-expressed). The expression levels of miR124 in J82 and T24 cell lines were decreased by $30 \%$ and $28 \%$, respectively, compared with normal urothelium cell line. UHRF1 plays an oncogenic role in bladder cancer and UHRF1 knockdown represses cell proliferation, invasion, migration and vasculogenic mimicry, while restoration of UHRF1 cancels the action induced by miR-124 in tumor cells. UHRF1 has a role in potentiating bladder cancer with higher rates of cell growth, metastasis and angiogenesis and its ectopic expression stimulates carcinogenesis by stimulating DNA methylation and histone suppressive changes of the suppressor gene, while UHRF1 decrease induces the stimulation and DNA demethylation of the suppressor gene promoter [19].

Caveolin 1 (CAV1) is a structure protein found within plasma membranes of various cell types. In a study on tumor tissue samples and adjacent normal tissues obtained from 73 patients with bladder cancer, along with bladder cancer cell lines (HT-1376, 5637, T24) and normal urothelium cell lines (SV-HUC-1), CAV1 was observed to be over-expressed in high-grade bladder cancer and was identified as a target of miR-124 which down-regulates CAV1 expression by linking to the 3'-UTR of CAV1 mRNA [20]. Under-expression of miR-124 in bladder cancer tissues and bladder cancer cell lines, compared with adjacent normal tissues and normal cell line, was also observed in this study. CAV1 binds integrin subunits to tyrosine kinase FYN, which is the first stage in the promotion of cell cycle progression. CAV1 expression was observed to be correlated with tumor grade and squamous cell differentiation. Over-expression of miR-124 inhibits the aggression of bladder cancer, while over-expression of CAV1 impairs the suppressive effects of miR-124 in malignant tissues [20].

Endothelin receptor type B (EDNRB) is a G-protein-linked receptor for endothelin 1 which had been associated with cell proliferation, apoptosis, and migration. One study that used tumor and normal adjacent tissues obtained from 27 bladder cancer patients, benign tissues obtained from 20 age and sex matched patients along with normal urothelium cell line (SV-HUC-1) and bladder cancer cell lines (TCCSUP, J82, 5637) showed that EDNRB mRNA is over-expressed and miR-124 is under-expressed in bladder cancer tissue and bladder cancer cell lines compared with normal urothelium tissue and cell line [21]. MiR-124-3p down-regulates EDNRB expression in bladder cancer cells by binding to EDNRB's 3'-UTR site. Transfecting of miR-124-3p mimics led to the down-regulation of EDNRB in tumor cells, while EDNRB expression was stimulated in bladder cancer tissues transfected with miR-124-3p inhibitors. Targeting EDNRB is another proven axis for miR-124-3p's regulation on proliferation or apoptosis in bladder cancer cells [21].

Metastasis-associated lung adenocarcinoma transcript 1 (MALAT1) is a long non-coding RNA that promotes proliferation and migration of bladder cancer cells. Jiao et al. found that MALAT1 can act as a ceRNA for miR-124, sinking its expression [22]. Foxq1 (Forkhead box protein Q1) is a protein that plays an important role in cell differentiation. The authors used 56 pairs of bladder cancer tissue and adjacent normal urothelium together with bladder cancer cell lines (T24, BIU-87, HEK293T), but without using normal urothelium cell lines. The article mentions that over-expression of miR124 in bladder cell lines leads to the inhibition of MALAT1 and foxq1 expression. Their study also found a clinical correlation between miR124 levels and survival, patients in the low expression group having a significantly worse survival than patients with high miR-124 expression [22].

$\mathrm{Xu}$ et al. studied the effect of miR-124-3p on the Rho-associated coiled-coil containing protein 
kinase 1 (ROCK1), using paired tumor tissue and adjacent normal urothelium obtained from 13 patients treated by radical cystectomy, along with tumor cell lines (T24, UM-UC-3, J82 and HEK293T) and normal urothelium cell line (SVHUC-1) [23]. Their results show that miR-124$3 \mathrm{p}$ has an important function in the epithelial mesenchymal transfer and regulates the expression of the genes that are responsible for cancer migration by directly targeting ROCK 13 '-UTR. ROCK 1 is over-expressed in bladder cancer tissues affecting cell migration and invasion. Silencing of ROCK1 caused the suppression of the migratory and invasive capability of bladder cancer cell in the same way the over-expression of miR-124-3p did [23].

There is also evidence that miR-124 regulates the activity of different cancer cells including bladder cancer. A study that was published in 2018 [24] and used bladder cancer cell lines (T24, UM-UC-3, SW780, HT1376, RT4, J82) and normal urothelium lines (SV-HUC-1, HEK293), without using patient bladder specimens, concludes that transfection of miR-124 in bladder cancer tissue leads to down-regulation of signal transducer and activator of transcription 3 (STAT3), a transcription factor that is normally over-expressed in bladder cancer cells. This process leads to the inhibition of tumor cell proliferation and migration by promoting apoptosis.

Mir-124 can also be measured in urine and can be a useful tool for predicting recurrence and survival of patients with bladder cancer. A research group from Japan [25] published an article in 2012 that investigated the alterations of micro RNA expressions in bladder cancer. The authors included 83 bladder cancer specimens and adjacent and distant normal urothelium obtained from patients treated with TUR-BT (trans-urethral resection of bladder tumor) and radical cystectomy, together with 6 specimens of normal urothelium collected from patients with renal cell carcinoma treated with nephrec- tomy, bladder cancer cell lines (T24, UM-UC-3, HT-1197, HT-1376, SW780, 5637) and normal bladder cell line (SV-HUC-1). Self-voided urine samples were obtained from 86 patients with bladder cancer and from 20 patients who were not diagnosed with cancer. Thirty-six urine specimens were also collected from patients who underwent TUR-BT, at 3-10 days postoperatively. The authors examined the expressions of 664 miRNAs in all types of bladder cancer cell lines and observed that only 12 miRNAs were highly methylated in multiple bladder cancer cell lines. They next examined the expression of the 12 miRNAs in normal urothelium and normal bladder cell line and distinguished only 4 miRNAs (miR-9-3, miR-124-2, miR-124-3 and miR-137) with low methylation levels in normal urothelium. High levels of miRNA methylation $(>15 \%)$ were found in bladder cancer tissues compared with normal bladder tissues, while adjacent tissues had higher methylation levels compared with distant tissues. Evaluation of urine specimens found that all four selected miRNAs were highly methylated in samples collected from bladder cancer patients, compared with samples collected from cancer-free patients. Important correlations were made between methylation level in urine specimens and expressions of the 4 miRNAs in bladder cancer tissues. Moreover, postoperative urinary methylation levels dropped after tumor resection compared with preoperative urinary methylation levels. Using ROC curve analysis, the authors established cutoff values for the 4 miRNAs and concluded that urinary methylation levels could act as a diagnostic test for bladder cancer [25].

The same research group published another study in 2017 that included 132 patients with a history of non-muscle-invasive bladder cancer treated with TUR-BT, used self-voided urine samples obtained before the control cystoscopy to determine methylation of the same 4 different miRNA genes (miR-9-3, miR-124-2, miR-124-3 
and miR-137) [26]. The authors found that recurrence was more common in patients with higher urine methylation levels, while methylation was independently associated with current or late recurrence. Important difference was noticed between the recurrence-free survival rates determined at one year after TUR-BT, for high methylation and low methylation groups $-66.7 \%$ and $94.3 \%$, respectively [26]. The article suggests that high urinary methylation levels is a marker for disease progression and can identify patients with non-muscle-invasive bladder cancer who benefit from early cystectomy. Regarding the pathological findings, urinary methylation was not correlated with tumor stage and grade [26]. Despite the advances made in oncological treatment and diagnosis, bladder cancer has high incidence, high prevalence, high morbidity, and high recurrence rates and does not have an available biomarker for better diagnosis or prognostic classification. A growing number of articles suggests that miRNAs contribute to bladder cancer development, bladder cancer cell invasion, angiogenesis, and metastasis. Studies have shown that bladder cancer tissues and cell lines contain low levels of miR-124 expression compared with normal urothelium. The selected articles that examined tissue miRNA showed different pathway of action for miR-124 and most of them analyzed the relation between the miRNA and its target and the way their expression change depending on the levels of the target and vice versa.

To summarize, higher expression of miR-124 can influence several cellular or molecular processes, including DNA methylation by suppressing DNMT3B and UHRF1, cell division and proliferation by suppressing CDK4, AURKA, and RDNRB, tumor angiogenesis by targeting VEGF and ROCK1, gene transcription together with tumor invasion and metastasis by regulating the XIST/AR axis, cell adhesion to extra-cellular matrix by suppressing ITGA3, cell cycle progression by suppressing CAV1 and cell proliferation and metastasis by silencing ROCK1, STAT3 and the MALAT1/foxq1 axis (Table 1). Thus, miR-124 can be considered an important anti-tumor miRNA in bladder cancer. One study even correlated the expression level of miR-124 with the survival of the patients with bladder cancer [22], assigning it a role as a prognostic biomarker.

High expression of miR-124 has shown an important apoptosis effect on different types of cancer cells, including bladder cancer. One study showed that overexpression of miR-124 could induce apoptosis in non-small-cell lung cancer patients and, furthermore, could lower the proliferation rates and enhance apoptosis of tumor cells after radiotherapy by targeting STAT3 [27]. Another study found that sulforaphane can activate miR-124 and increase its levels in patients with gastric cancer. MiR-124 targets the interleukin-6 receptor and STAT3 to control the cancer stem cells and, consecutively, to reduce metastasis and improve chemotherapy outcomes [28]. Thus, miR-124 can be an important therapeutic target for oncological treatment of bladder cancer, improving efficacy of chemotherapy and radiotherapy by acting like a therapy modulator. Methylation levels of miR-124 in urine is useful for identifying patients with bladder cancer [25]. Furthermore, high urinary methylation levels of miR-124 can identify patients with a high risk for recurrence [26]. Thus, urinary miR-124 can play an important role as a diagnostic and prognostic biomarker.

FDA approved the use of the first two RNA interference drugs in 2018 and 2020 and, consequently, more and more types of RNA drugs entered clinical trials. Patisiran was approved in 2018 for treating polyneuropathy caused by hereditary transthyretin-mediated amyloidosis, while Givosiran was authorized in acute hepatic porphyria treatment. 
Table 1. Authors that identified targets of miR-124 in bladder cancer and the cell lines they used

\begin{tabular}{|c|c|c|c|c|}
\hline Author & $\begin{array}{l}\text { Number of } \\
\text { cases }\end{array}$ & $\begin{array}{l}\text { Bladder cancer cell lines } \\
\text { used }\end{array}$ & $\begin{array}{l}\text { Normal urothelium } \\
\text { cell line used }\end{array}$ & Target of miR-124 \\
\hline $\mathrm{Xu}(2013)[23]$ & 13 patients & $\begin{array}{l}\text { T24, J82, UM-UC-3, } \\
\text { HEK293T }\end{array}$ & 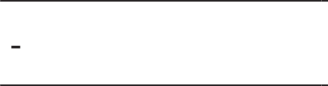 & ROCK1 \\
\hline Zhang (2014) [12] & 27 patients* & $\begin{array}{l}\text { HT1197, J82, HT1376, } \\
5637\end{array}$ & HBEC & CDK4 \\
\hline Wang X (2015) [19] & 15 patients & T24, J82 & SV-HUC-1 & UHRF1 \\
\hline Xiong (2017) [17] & 67 patients & $\begin{array}{ll}\text { TCCSUP, } & \text { EJ, } \\
\text { UM-UC-3 }\end{array}$ & SV-HUC-1 & XIST, AR \\
\hline Yuan (2017) [15] & 36 patients & T24, 5637, J82, RT4 & SV-HUC-1 & AURKA \\
\hline Wang JR (2018) [18] & 36 patients & $\mathrm{T} 24,5637, \mathrm{~J} 82$ & SV-HUC-1 & ITGA3 \\
\hline Zhou (2018) [20] & 73 patients & T24, 5637, HT1376 & SV-HUC-1 & CAV1 \\
\hline Zo (2018) [11] & 84 patients & T24, 5637, RT4 & SV-HUC-1 & DNMT3B \\
\hline Jiao (2018) [22] & 56 patients & T24, BIU-87, HEK293T & - & MALAT1, foxq1 \\
\hline Wang S (2018) [24] & - & $\begin{array}{l}\text { T24，UM-UC-3，SW780, } \\
\text { HT1376, RT4, J82 }\end{array}$ & SV-HUC-1, HEK293 & STAT3 \\
\hline Cao (2019) [13] & 83 patients & $\begin{array}{l}\text { T24, 5637, J82, } \\
\text { UM-UC-2 }\end{array}$ & SV-HUC-1 & $\begin{array}{l}\text { CDK4, E2F3, Ki-67, } \\
\text { VEGF }\end{array}$ \\
\hline $\mathrm{Fu}(2019)[21]$ & 27 patients $* *$ & TCCSUP, 5637, J82 & SV-HUC-1 & EDNRB \\
\hline
\end{tabular}

${ }^{(*)}$ Authors did not use adjacent normal urothelium obtained from bladder cancer patients

${ }^{(* *)}$ Authors collected 20 benign tissues from age and sex matched patients

Further research in the modern genomics and molecular biology era can lead to establish miR124 as a biomarker for bladder cancer with an important role in diagnosis, recurrence, prognosis and survival of the patients.

Provided that every type of cancer has a different pattern of miRNA expression, bladder cancer has constantly shown under-expression of miR124 in tumor cells and high methylation levels in urine. Therefore, it can be a useful tool for establishing early diagnosis for bladder cancer patients, for including patients in prognostic groups and for developing targeted molecular therapy and personalized treatment. Still, the effective contribution and particular bio-chemical processes between miR-124 and bladder cancer are not completely detailed and require more research. This should be the first step that scientists should take in order to elucidate the complete cellular processes in which miR-124 may be involved. It is also important to establish the role of miR-124 as a modulator or even as a proper treatment for patients undergoing chemotherapy and radiotherapy, similarly to the way it was studied for other types of cancer, in order to obtain better treatment outcomes. Thus, future perspectives should also include increasing the level of miR-124 expression in tumor tissue for patients with bladder cancer, hoping that this therapeutic strategy can change the way we treat this disease.

\section{References}

1. Globocan 2018, International Agency for Research on Cancer, World Health Organization, 2019 Jan. Available from http://gco.iarc.fr.

2. Dyer T, Siemens DR, Nippak P, Meyer J, Booth CM. Histology at transurethral resection of bladder tumor and radical cystectomy for bladder cancer: Insights from population-based data. Can Urol Assoc J. 2020 Sep 28. DOI: 10.5489/cuaj.6856

3. Lagos-Quintana M, Rauhut R, Yalcin A, Meyer J, Lendeckel W, Tuschl T. Identification of tissue-specific microRNAs from mouse. Curr Biol. 2002 Apr 
30;12(9):735-9. DOI: 10.1016/S0960-9822(02)008096

4. Ben Gacem R, Ben Abdelkrim O, Ziadi S, Ben Dhiab M, Trimeche M. Methylation of miR-124a-1, miR124a-2, and miR-124a-3 genes correlates with aggressive and advanced breast cancer disease. Tumour Biol. 2014 May;35(5):4047-56. DOI: 10.1007/s13277-0131530-4

5. Chen ZY, Wang XY, Yang YM, Wu MH, Yang L, Jiang DT, Cai H, Peng Y. LncRNA SNHG16 promotes colorectal cancer cell proliferation, migration, and epithelial-mesenchymal transition through miR-1243p/MCP-1. Gene Ther. 2020 Aug 28. DOI: 10.1038/ s41434-020-0176-2

6. Liu T, Zhu J, Du W, Ning W, Zhang Y, Zeng Y, Liu $Z$, Huang JA. AKT2 drives cancer progression and is negatively modulated by miR-124 in human lung adenocarcinoma. Respir Res. 2020 Sep 1;21(1):227. DOI: 10.1186/s12931-020-01491-0

7. Yang T, Jin X, Lan J, Wang W. Long non-coding RNA SNHG16 has Tumor suppressing effect in acute lymphoblastic leukemia by inverse interaction on hsa-miR124-3p. IUBMB Life. 2019 Jan;71(1):134-42. DOI: 10.1002/iub. 1947

8. Chen XX, Lin J, Qian J, Qian W, Yang J, Ma JC, et al. Dysregulation of miR-124-1 predicts favorable prognosis in acute myeloid leukemia. Clin Biochem. 2014 Jan;47(1-2):63-6. DOI: 10.1016/j.clinbiochem.2013.09.020

9. Li Y, Yan J, Wang Y, Wang C, Zhang C, Li G. LINC00240 promotes gastric cancer cell proliferation, migration and EMT via the miR-124-3p / DNMT3B axis. Cell Biochem Funct. 2020;1-10.

10. Moghadasi M, Alivand M, Fardi M, Moghadam KS, Solali S. Emerging molecular functions of microRNA-124: Cancer pathology and therapeutic implications. Pathol Res Pract. 2020 Mar;216(3):152827. DOI: 10.1016/j.prp.2020.152827

11. Zo RB, Long Z.: MiR-124-3p suppresses bladder cancer by targeting DNA methyltransferase 3B. J Cell Physiol. 2018;1-11. DOI: 10.1002/jcp.26591

12. Zhang T, Wang J, Chang J et al.: MiR-124 retards bladder cancer growth by directly targeting CDK4. Acta Biochim Biophys Sin (Shanghai) 2014 Dec;46(12):1072-9. DOI: $10.1093 / \mathrm{abbs} / \mathrm{gmu} 105$

13. Cao Z, Xu L, Zhao S, Zhu X. The functions of microRNA-124 on bladder cancer. Onco Targets Ther. 2019;12:3429-39. DOI: 10.2147/OTT.S193661

14. Qu Y, Huang X, Li Z, Liu J, Wu J, Chen D, et al. miR199a-3p inhibits aurora kinase $A$ and attenuates prostate cancer growth: new avenue for prostate cancer treatment. Am J Pathol. 2014 May;184(5):1541-9. DOI: 10.1016/j.ajpath.2014.01.017

15. Yuan Q, Sun T, Ye F, Kong W, Jin H. MicroRNA-124-3p affects proliferation, migration and apoptosis of bladder cancer cells through targeting AURKA. Cancer Biomark. 2017;19(1):93-101. DOI: 10.3233/CBM-160427

16. Lombard AP, Mudryj M. The emerging role of the androgen receptor in bladder cancer. Endocrin Rel Cancer. 2015;22. DOI: 10.1530/ERC-15-0209

17. Xiong Y, Wang L, Li Y, Chen M, He W, Qi L. The Long Non-Coding RNA XIST Interacted with MiR124 to Modulate Bladder Cancer Growth, Invasion and Migration by Targeting Androgen Receptor (AR). Cell Physiol Biochem. 2017;43(1):405-18. DOI: 10.1159/000480419

18. Wang JR, Liu B, Zhou L, Huang YX. MicroRNA-124-3p suppresses cell migration and invasion by targeting ITGA3 signaling in bladder cancer. Cancer Biomark. 2019;24(2):159-72. DOI: 10.3233/CBM182000

19. Wang X, Wu Q, Xu B, Wang P, Fan W, Cai Y, Gu X, Meng F. MiR-124 exerts tumor suppressive functions on the cell proliferation, motility and angiogenesis of bladder cancer by fine-tuning UHRF1. FEBS J. 2015 Nov;282(22):4376-88. DOI: 10.1111/febs.13502

20. Zhou W, He L, Dai Y, Zhang Y, Wang J, Liu B. MicroRNA-124 inhibits cell proliferation, invasion and migration by targeting CAV1 in bladder cancer. Exp Ther Med. 2018 Oct;16(4):2811-20. DOI: 10.3892/ etm.2018.6537

21. Fu W, Wu X, Yang Z, Mi H. The effect of miR-124-3p on cell proliferation and apoptosis in bladder cancer by targeting EDNRB. Arch Med Sci. 2019 Sep;15(5):115462. DOI: 10.5114 /aoms. 2018.78743

22. Jiao D, Li Z, Zhu M, Wang Y, Wu G, Han X. LncRNA MALAT1 promotes tumor growth and metastasis by targeting miR-124/foxq1 in bladder transitional cell carcinoma (BTCC). Am J Cancer Res. 2018;8(4):74860.

23. Xu X, Li S, Lin Y, Chen H, Hu Z, Mao Y, et al. MicroRNA-124-3p inhibits cell migration and invasion in bladder cancer cells by targeting ROCK1. J Transl Med. 2013 Nov 2;11:276. DOI: 10.1186/1479-5876$11-276$

24. Wang S, Wu G, Han Y, et al. miR-124 regulates STAT3-mediated cell proliferation, migration and apoptosis in bladder cancer. Oncol Lett. 2018;16(5):587581. DOI: $10.3892 / \mathrm{ol} .2018 .9341$

25. Shimizu T, Suzuki H, Nojima M, et al. Methylation of a panel of microRNA genes is a novel biomarker for detection of bladder cancer. Eur Urol. 2013 Jun;63(6):1091100. DOI: 10.1016/j.eururo.2012.11.030

26. Shindo T, Shimizu T, Nojima M, Niinuma T, Maruyama $\mathrm{R}$, Kitajima H, et al. Evaluation of Urinary DNA Methylation as a Marker for Recurrent Bladder Cancer: A 2-Center Prospective Study. Urology. 2018 Mar; 113:71-8. DOI: 10.1016/j.urology.2017.11.025

27. Wang M, Meng B, Liu Y, Yu J, Chen Q, Liu Y. MiR124 Inhibits Growth and Enhances Radiation-Induced 
Apoptosis in Non-Small Cell Lung Cancer by Inhibiting STAT3. Cell Physiol Biochem. 2017;44(5):201728. DOI: $10.1159 / 000485907$

28. Wang X, Li Y, Dai Y, Liu Q, Ning S,Liu J, et al. Sul- foraphane improves chemotherapy efficacy by targeting cancer stem cell-like properties via the miR-124/ IL-6R/STAT3 axis. 2016;Sci Rep 6:36796(2016). DOI: $10.1038 /$ srep36796 